\title{
Malignant mesothelioma presenting as a breast mass
}

\author{
${ }^{1} \mathrm{~A}$ Aujayeb, ${ }^{2} \mathrm{H}$ Clague, ${ }^{3} \mathrm{~S}$ Pollock, ${ }^{4} \mathrm{~S}$ Stamenkovic, ${ }^{5} \mathrm{~A}$ Hughes \\ ${ }^{1}$ Specialist Registrar, ${ }^{2}$ Consultant Physician; ${ }^{3}$ Lung Cancer Nurse Specialist; Respiratory Medicine Department, Sunderland Royal Hospital, \\ Sunderland; ${ }^{4}$ Consultant Thoracic Surgeon; ${ }^{5}$ Consultant Medical Oncologist; Freeman Hospital, Newcastle upon Tyne, UK
}

\begin{abstract}
We present a case of a male patient with a breast mass, found to be a malignant mesothelioma. We discuss the diagnostic challenges, the need for heightened awareness in suspected cases, the histological classification of mesotheliomas and the treatments available. We believe this to be the second reported case of a mesothelioma presenting as a breast mass.
\end{abstract}

KEYWORDS Breast, mesothelioma, cancer, epithelioid, sarcomatoid

\author{
Correspondence to A Aujayeb, \\ Repiratory Medicine Department, \\ Sunderland Royal Hospital, \\ Kayll Road, Sunderland \\ SR4 7TP, UK
}

tel. +44 (0)778 65 I 280 I

e-mail aujayeb@doctors.net.uk

DECLARATION OF INTERESTS No conflicts of interest declared.

\section{CASE REPORT}

A 60-year-old male presented with suspected breast cancer. He had a medical history of hypertension, learning difficulties and previous alcohol abuse. The patient had no family history of breast cancer. He was an ex-smoker ( 15 pack-years history) and worked as a labourer, with no reported exposure to asbestos.

On examination, we identified a non-tender $13 \mathrm{~cm}$ size mass in the right breast, invading the pectoralis muscle and extending into the axillary tail (Figures $I$ and 2). Dilated veins showed on the surrounding skin. A transthoracic ultrasound and two core biopsies were performed. The ultrasound suggested an intrathoracic origin and the core biopsies showed extensive infiltration by tumour cells. Potential diagnoses included mesothelioma, poorly differentiated carcinoma or primary lung carcinoma.

A full staging computed tomography (CT) scan was performed to measure the degree to which the cancer had spread (Figures 3 and 4). The scan showed a $14 \mathrm{~cm}$ mass in the right breast, straddling the chest wall, with a small tissue component in the anterior right pleura. There was also a large right pleural effusion with two soft tissue nodules on the pleura of the mediastinal interface.

The patient was referred for a surgical incisional biopsy. The pleural effusion was not drained during this procedure because there were minimal symptoms. However the patient's dyspnoea progressed and a posterior, single port, video-assisted thoracic surgery (VATS) procedure was carried out, draining 4.8 litres of blood-stained fluid from the area. It was clear on visual inspection that there was a large intrathoracic component of the tumour involving both visceral and parietal pleura. The right lung failed to expand, so a long-term intrapleural catheter (@Rocket) was put in place.
Histology showed a tumour with a solid nested growth pattern and epithelioid cells with abundant cytoplasm, pleomorphic nuclei and prominent, multiple nucleoli with mitotic figures. The tumour cells show no evidence of mucin production. Immunohistochemistry showed strong positive expression with calretinin (a calcium-binding protein detected in most malignant mesotheliomas [MM]), epithelial membrane antigen (an established serum marker for breast cancer), Wilms tumour I (WTI), an independent marker of breast cancer, pan-cytokeratin (a traditional non-specific epithelial marker),thrombomodulin (a naturally occurring anticoagulant that may play a role in tumour metastases), D2-40 (an antibody used in identifying mesothelioma), e-cadherin (a protein found on the surface of breast cancer cells) and cytokeratin 5, 6 and 7 . Cytokeratin 5/6 (CK 5/6) immunoreactivity has been observed in the vast majority of cases of MM but only rarely in pulmonary adenocarcinomas. Therefore CK 5/6 has been used to distinguish MM from pulmonary adenocarcinoma in which CK 7 is observed predominantly (Figures 5 and 6).

The most likely diagnosis was epithelioid mesothelioma as the morphological appearances fit and this diagnosis is fully supported by positive immunostaining with calretinin, cytokeratin 5/6, D2-40, thrombomodulin and WTI. Chemotherapy was not possible, due to the patient's condition. Palliative radiotherapy was considered for pain control but the patient's health deteriorated and he died before treatment could be initiated.

\section{DISCUSSION}

\section{Epidemiology and aetiology}

Malignant mesothelioma is a rare form of cancer that develops in the pleura or peritoneal serosa (a membrane that encloses several body cavities). The majority of cases are associated with exposure to asbestos, although the exposure dose is often lower in cases of mesothelioma than for asbestosis or an asbestos-related lung cancer. There is typically a delay between exposure and development of the disease. Asbestos use was prevalent in 

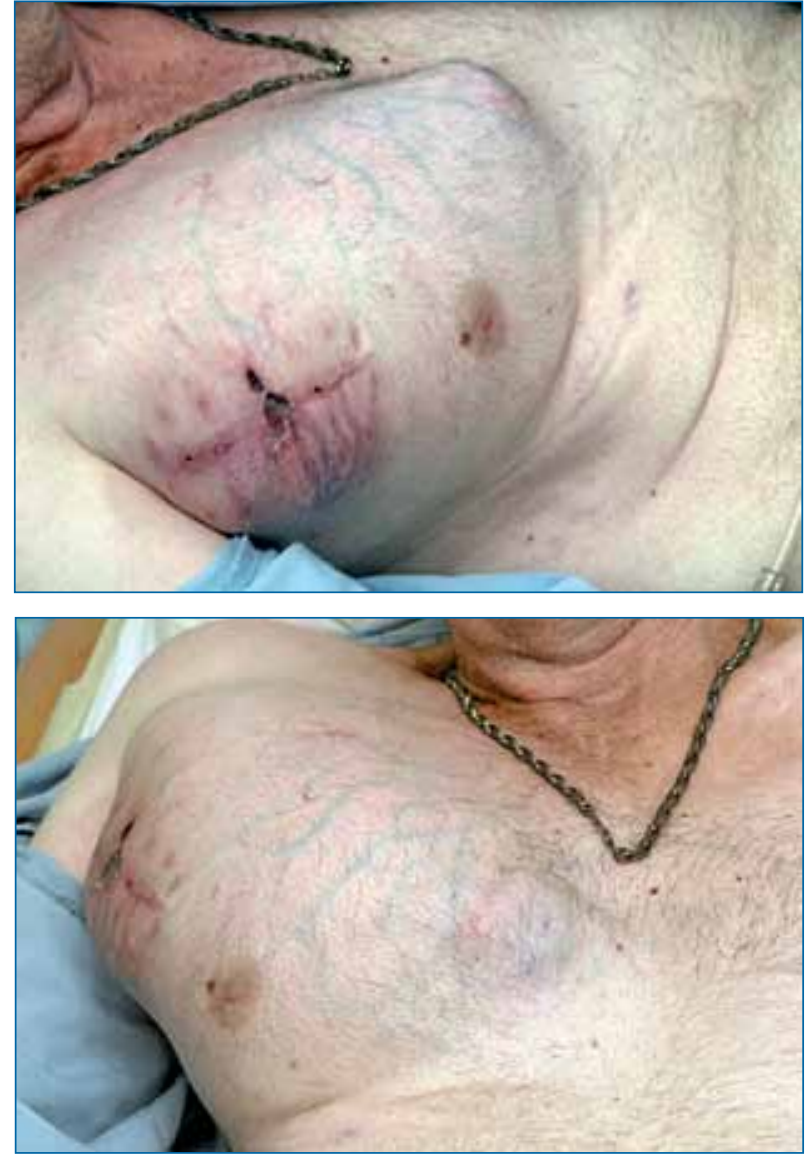

FIGURES I AND 2 Large interior chest wall/breast mass with ulceration at the site of biopsy.

the UK in the 1960s and 1970s, especially in the north east of England, where our patient lived and worked. A 1999 study predicted an epidemic of mesothelioma in Europe, with an estimated quarter of a million deaths in Western Europe between 1999 and 2034.' More recent figures from the Health and Safety Executive (a national independent watchdog for work-related health, safety and illness) show that yearly mortality from MM in the UK has risen from 158 in 1968 to 2,249 in $2008 .{ }^{2}$ The majority of cases are in men. By 2050, around 91,000 deaths are predicted, with approximately 61,000 from 2007 onwards. ${ }^{2,3}$

The lack of exposure history in some patients, and the fact that only a small proportion of exposed individuals are affected has led some to suggest other aetiological factors such as a genetic predisposition, or exposure to the tumour-inducing simian vacuolating virus 40 (SV40). The gene sequence of that virus has been identified in over $50 \%$ of patients with epithelioid mesotheliomas. However, epidemiologic studies of patients with virus inoculation through contaminated polio vaccines have not shown a strong link. ${ }^{3-5}$

\section{Presentations of malignant mesothelioma}

Lee et al. ${ }^{6}$ first described a similar case in a female patient presenting with a breast mass. Malignant mesothelioma has also been reported to present as sclerosing

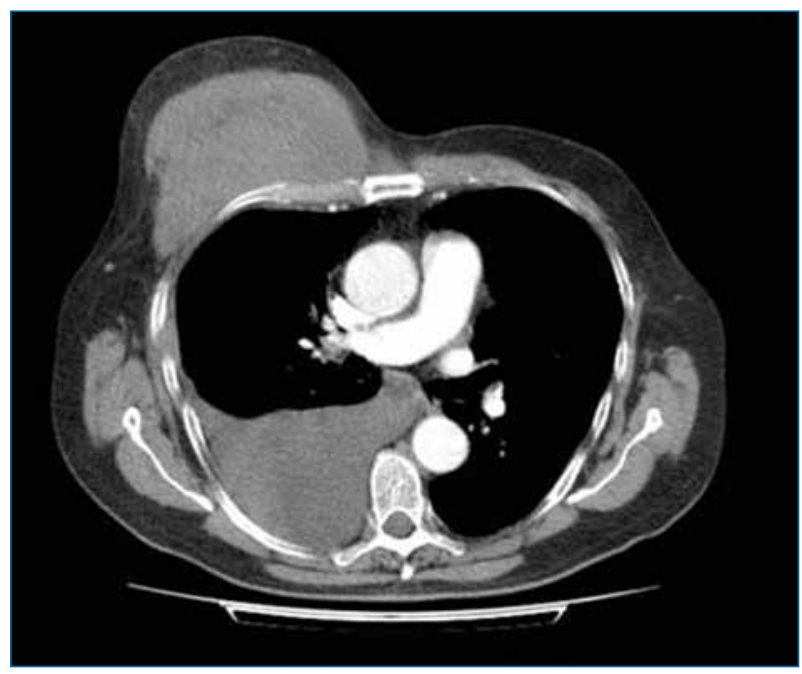

FIGURE 3 Computed tomography (CT) scan showing a $14 \mathrm{~cm}$ mass in the right breast invading the chest wall with a small tissue component in the anterior right pleura with a large right pleural effusion.

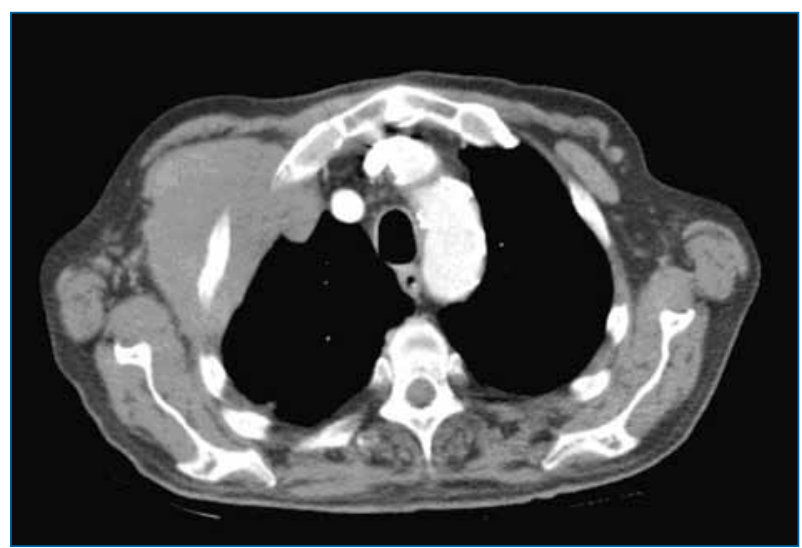

FIGURE 4 Computed tomography (CT) scan showing a right-sided soft tissue chest wall mass, of probable pleural origin, with adjacent axillary and subclavian lymphadenopathy and possible rib destruction.

mediastinitis, ${ }^{7}$ an intrascrotal mass, ${ }^{8}$ pericardial disease, ${ }^{9}$ and with meningeal and brain metastases. ${ }^{10}$

The majority of MM are pleural. Symptoms include a cough, dyspnoea, weight loss and chest pain. The features of $\mathrm{MM}$ are circumferential nodular lung encasement, pleural thickening with irregular pleuro-pulmonary margins, pleural thickening with superimposed nodules and the extension of pleural thickening onto the mediastinal surface." It is difficult to differentiate irregular pleural thickening from pleural effusions on plain chest radiographs so a CT scan is the most effective way of distinguishing them.

\section{Pathology}

Malignant mesothelioma can often present initially as pleural effusions. The use of cytology for diagnosis can be difficult as examination of pleural fluid for MM can have an overall sensitivity of just $32 \%^{12}$ and the sensitivity can be as 


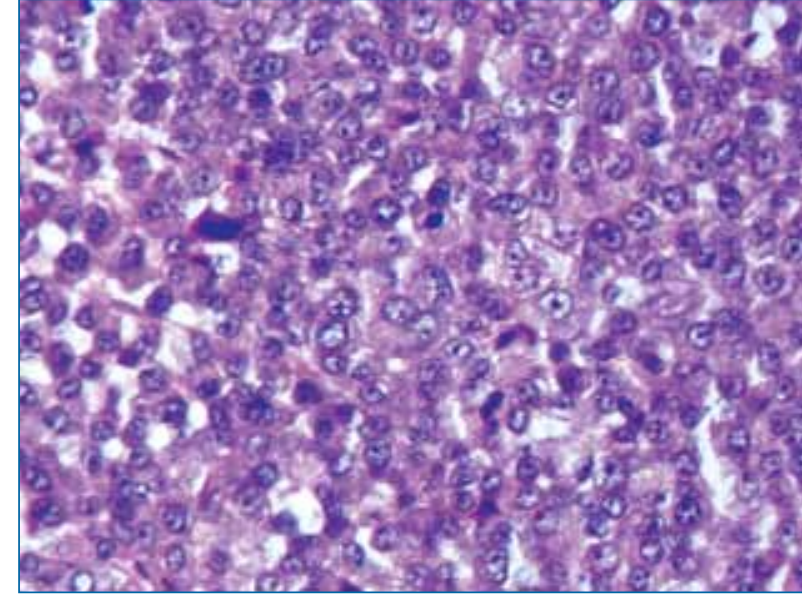

FIGURE 5 Haematoxylin and eosin at a magnification $\times 200$ showing a solid proliferation of atypical polygonal cells with amphophilic cytoplasm and large irregular nuclei containing conspicuous nucleoli. Apoptotic bodies and mitotic activity are also present.

low as $20 \%$ in sarcomatoid MM. ${ }^{13}$ Blind biopsy techniques are quick to perform and inexpensive. However, Abrams' punch pleural biopsy is less effective at diagnosing pleural thickening than a CT-guided percutaneous pleural biopsy and is therefore not ideal. Other techniques include ultrasound-guided or surgical video-assisted thoracoscopic biopsy. In a review of 45 cases of MM, open pleural biopsy had a sensitivity of $95 \%$ and specificity of $100 \%$ for definitive diagnosis. Closed blind biopsies had a sensitivity of $16 \%$ and a specificity of $94 \% .{ }^{14}$ All CT-guided biopsies confirmed mesothelioma but there were only five cases. In another study of 100 cases of $\mathrm{MM}$, the sensitivity of all CT-guided core biopsies is quoted as $86 \%{ }^{15}$ In that study, needle track seeding, which is a potential hazard for any biopsy, had an incidence of $4 \%$ for image-guided coreneedle biopsy and $22 \%$ for surgical biopsy. The definitive procedure for confirmation of diagnosis should be decided upon in a cancer multidisciplinary meeting.

Histology is an important tool in establishing a definitive diagnosis of MM. There are three main histological subtypes of $\mathrm{MM}$, with differing prognoses: $50 \%$ of $\mathrm{MM}$ is diagnosed as epithelioid, $16 \%$ as sarcomatoid and $34 \%$ as both epithelioid and sarcomatoid. ${ }^{17}$

Pathological diagnosis is also challenging. The first step is to differentiate an epithelioid mesothelioma from a sarcomatoid mesothelioma or an adenocarcinoma and establish that the changes are not from local pleural reactivity. Histological examination alone is usually insufficient in making a correct diagnosis and most pathologists rely on a panel of immunohistochemical markers to help differentiate between these conditions, although none can distinguish absolutely between these two diseases with complete sensitivity and specificity.

Epithelioid MM and metastatic adenocarcinomas share some histological attributes and can be difficult to distinguish

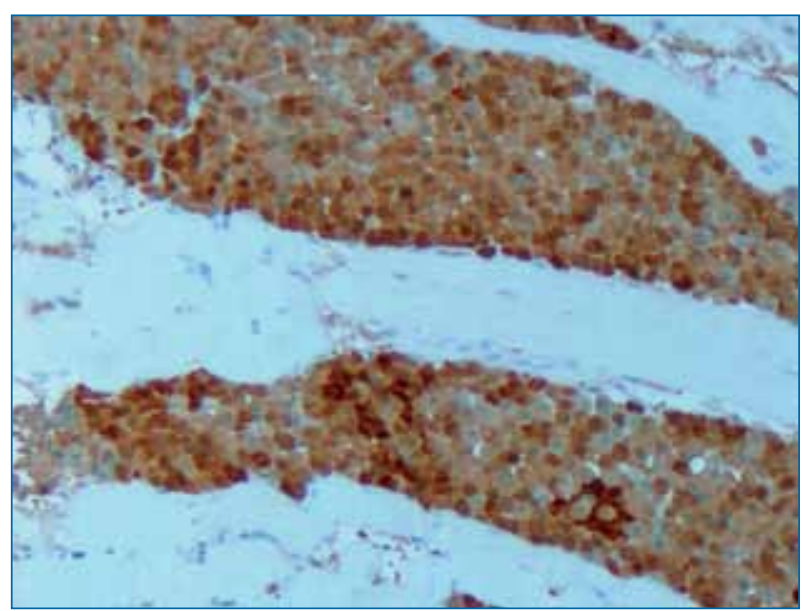

FIGURE 6 Immunostain at a magnification of $\times 200$ with calretinin showing expression in the cytoplasm and nuclei of the atypical mesothelial cells infiltrating with a solid pattern among the muscle fibres of the chest wall.

from each another. Epithelioid MM stain for calretinin, CK5/6,WTI, thrombomodulin, $n$-cadherin and vimentin. ${ }^{16}$ Pulmonary adenocarcinomas are negative for those but positive for carcinoembryonic antigen (84\%), CDI5 (77\%), Ber-EP-4 (82\%), TTF-I, e-cadherin and MOC-3I. ${ }^{17}$ It is worth noting that histochemical stains such as periodic acid-Schiff tend to be positive in both. Non-pulmonary adenocarcinomas can show different expression which is why these stains need to be assessed in combination with the clinical-radiological picture. ${ }^{17}$

The cells in sarcomatoid MM tend to be elongated spindle-shaped cells. They are irregular and overlap, in contrast to epithelioid MM where the cells are welldefined and uniform in shape. Sarcomatoid MM are also usually negative for TTF-I, CK5/6 and WTI. Pancytokeratin stains $70 \%$ of sarcomatoid mesotheliomas but also $17 \%$ of sarcomas and $90 \%$ of sarcomatoid carcinomas, and $100 \%$ of epithelioid mesotheliomas. Calretinin and thrombomodulin also stain $70 \%$ of sarcomatoid MM. ${ }^{16}$

The morphology of the cells and the stains as described in our case report confirmed the diagnosis for our patient.

\section{Treatment options}

The standard treatment in patients who have a World Health Organization performance status of 0 or 1 (restricted in strenuous activity but able to move around and carry out light work) is palliative chemo-therapy. ${ }^{18}$ The Evaluation of Mesothelioma in a Phase III trial of Pemetrexed with Cisplatin (EMPHACIS) study was a single-blind, international, multicentre trial comparing cisplatin and pemetrexed with cisplatin alone, in 448 patients. ${ }^{19}$ Combination chemotherapy was found to be superior, with a median survival of 12 .I months vs 9.3 months $(p=0.02)$. Response rates and rate of progression were also superior for the combination group. ${ }^{19}$ This trial led to pemetrexed becoming the only 
licensed drug for the treatment of mesothelioma. Further work has shown that the addition of vitamin supplementation reduces the toxicity of pemetrexed without reducing the efficacy. This was also demonstrated in the EMPHACIS trial. Ongoing trials are now examining different therapies in the first-line setting and second-line treatment.

Surgical treatments are rarely used. Extrapleural pneumonectomies (EPP) for example, involve the removal of as much macroscopic disease as possible. A systematic review in 2005 concluded that the role of EPP could not be defined..$^{20}$ Although a recent UK multimodality pilot study (Mesothelioma and Radical Surgery [MARS] I) ${ }^{21}$ was carried out in preparation for a phase II trial, there are no randomised controlled trials of EPP. Video assisted thoracoscopic surgery (VATS) pleurectomy and decortication (a similar debulking operation) is a lung-sparing option, and is being considered for a future MARS 2 trial. A palliative operation such as VATS pleurectomy can prevent re-accumulation of effusions and has been linked with an increased survival rate ${ }^{22}$ but there have been no randomised trials to date. These operations are best performed on carefully selected patients with a good performance status and adequate cardiovascular reserve, in a multi-modality setting and as part of ongoing clinical trials.

Radiotherapy is used for symptom control but large doses cannot usually be administered due to the potential side-effects on the other mediastinal structures. Prophylactic radiotherapy is also sometimes applied to biopsy needle tracts to prevent local MM seeding along tract sites, but there has been no proven benefit. A 1995 study found that tumour seeding in tract sites was prevented in all 20 patients who received prophylactic radiotherapy. ${ }^{23}$ However, O'Rourke et al. ${ }^{24}$ devised a similar study involving $6 \mathrm{I}$ patients which indicated that radiotherapy made no statistical difference in the risk of subcutaneous tumour growth. However, $35 \%$ of their patients experienced significant sideeffects from the radiotherapy, re-igniting the debate as to whether radiotherapy should only be given if tumours appear.

Treatment of MM is therefore essentially palliative and should be focused on improving symptoms and prolonging life.

\section{CONCLUSION}

Mesothelioma is an increasingly common disease and the number of unusual presentations is therefore likely to rise. Mesothelioma spreads by direct invasion into surrounding structures and an intrathoracic origin should be considered in the differential diagnosis of unusual chest wall or breast masses. Adequate tissue sampling and immunohistochemical interpretation is essential to make a correct diagnosis but the risk of subsequent tumour seeding at instrumentation sites also needs to be considered. Diagnosis is improved by a multidisciplinary approach that includes both clinical and radiological correlation. Mesothelioma is invariably a fatal disease, although a combination of chemotherapy and radiotherapy in selected patients has been shown to control symptoms and improve survival rates.

\section{Acknowledgements}

Our sincere thanks go to $\mathrm{Dr} J \mathrm{~J}$ Majo and $\mathrm{Dr} \mathrm{R}$ Howitt for the pathology images and their interpretation.

\section{REFERENCES}

I Peto J, Decarli A, La Vecchia, C et al. The European mesothelioma epidemic. Br J Cancer 1999; 79:666-72. http://dx.doi.org//0.1038/ sj.bjc.6690I05

2 Health and Safety Executive (HSE). Mesothelioma [Internet]. London: HSE; [cited 20I I Aug 3I]. Available from: http://www.hse. gov.uk/statistics/causdis/mesothelioma/index.htm

3 Health and Safety Executive (HSE).RR728-Projection of mesothelioma mortality in Great Britain [Internet]. London: HSE; [cited 20II Aug 3I].Available from: http://www.hse.gov.uk/research/rrhtm/rr728.htm

4 Barbanti-Brodano G, Sabbioni S, Martini F et al. Simian virus 40 infection in humans and association with human diseases: results and hypotheses. Virology 2004; 3 18: I-9. http://dx.doi.org/10.1016/j. virol.2003.09.004

5 Strickler HD, Goedert JJ, Devesa SS et al. Trends in US pleural mesothelioma incidence rates following simian virus 40 contamination of early poliovirus vaccines.J Natl Cancer Inst 2003; 95:38-45. http://dx.doi.org//0.1093/jnci/95.I.38

6 Cutrone R, Lednicky J, Dunn G et al. Some oral poliovirus vaccines were contaminated with infectious SV40 after 196I. Cancer Res 2005; 65:I0273-9. http://dx.doi.org/I0.I I58/0008-5472.CAN-05-2028

7 Lee JC, Banerjee S, King DM. Breast or chest? A diagnostic conundrum. Br J Radiol 2005; 78:47I-2. http://dx.doi.org/I0.1259/ bjr/77/08289

8 Crotty TB, Colby TV, Gay PC et al. Desmoplastic malignant mesothelioma masquerading as sclerosing mediastinitis: a diagnostic dilemma. Hum Pathol 1992;23:79-82.http://dx.doi.org/I0.1016/00468I77(92)900I7-W

9 Takashi S, Takehiro S, Takanaeo T et al. Malignant mesothelioma of tunica vaginalis testis with atypical cells in aspirated fluid of hydrocele. Japanese Journal of Clinical Urology 2005; 59:4I 5-7.

10 Suman S, Schofield P, Large S. Primary pericardial mesothelioma presenting as pericardial constriction: a case report. Heart 2004; 90:e4. http://dx.doi.org/10.I I36/heart.90.I.e4

II Bohn U, Gonzalez L, Martin LM et al. Meningeal and brain metastases in primary malignant pericardial mesothelioma. Ann Oncol 1994; 5:660-I.

12 Metintas M, Ucgun I, Elbek $O$ et al. Computed tomography features in malignant pleural mesothelioma and other commonly seen pleural diseases. Eur J Radiol 2002; 4I:I-9. http://dx.doi.org/I0.1016/ S0720-048X(0I)00426-0

13 Renshaw AA, Dean BR, Antman $\mathrm{KH}$ et al. The role of cytologic evaluation of pleural fluid in the diagnosis of malignant mesothelioma. Chest 1997; II I:I06-9. http://dx.doi.org/I0.1378/chest. I II.I.106

I4 Rakha EA, Patil S, Abdulla K et al. The sensitivity of cytologic evaluation of pleural fluid in the diagnosis of malignant mesothelioma. Diagn Cytopathol 2010; 38:874-9. http://dx.doi.org/I0.1002/dc.2I303

I5 Attanoos RL, Gibbs AR. The comparative accuracy of different pleural biopsy techniques in the diagnosis of malignant mesothelioma. Histopathology 2008; 53:340-4. http://dx.doi. org/I0.IIII/j. I365-2559.2008.03099.x 
16 Agarwal PP, Seely JM, Matzinger FR et al. Pleural mesothelioma: sensitivity and incidence of needle track seeding after imageguided biopsy versus surgical biopsy. Radiology 2006; 241:589-94. http://dx.doi.org/I0.II48/radiol.24I205I020

17 Husain AN, Colby TV, Ordonez NG et al. Guidelines for pathologic diagnosis of malignant mesothelioma: a consensus statement from the international mesothelioma interest group. Archives of Pathology \& Laboratory Medicine 2009; |33:|3|7-3|.

18 Ordonez NG. The immunohistochemical diagnosis of mesothelioma: a comparative study of epithelioid mesothelioma and lung adenocarcinoma. Am J Surg Pathol 2003; 27:1031-5I. http://dx.doi. org/I0.1097/00000478-200308000-0000 I

19 Budde LS, Hanna NH. Pemetrexed (Alimta): improving outcomes in malignant pleural mesothelioma. Expert Rev Anticancer Ther 2004; 4:36 I-8. http://dx.doi.org//0.I586//4737|40.4.3.36I

20 Vogelzang NJ, Rusthoven JJ, Symanowski J et al. Phase III study of pemetrexed in combination with cisplatin versus cisplatin alone in patients with malignant pleural mesothelioma.J Clin Oncol 2003; 2 I: 2636-44. http://dx.doi.org/I0.1200/JCO.2003.II.I36
21 Maziak DE, Gagliardi A, Haynes AE et al. Surgical management of malignant pleural mesothelioma: a systematic review and evidence summary. Lung Cancer 2005; 48:157-69. http://dx.doi.org/10.1016/j. lungcan.2004.II.003

2I Treasure T, Tan C, Lang-Lazdunski L et al. The MARS trial: mesothelioma and radical surgery. Interact Cardiovasc Thorac Surg 2006; 5:58-9. http://dx.doi.org//0.15/0/icvts.2005.123430

22 Waller DA, Morritt GN, Forty J. Video-assisted thoracoscopic pleurectomy in the management of malignant pleural effusion. Chest 1995; 107: 1454-6. http://dx.doi.org/10.1378/chest. 107.5.1454

23 Low EM, Khoury GG, Matthews AW et al. Prevention of tumour seeding following thoracoscopy in mesothelioma by prophylactic radiotherapy. Clin Oncol (R Coll Radiol) 1995; 7:317-8. http://dx.doi. org/I0.I016/S0936-6555(05)80540-0

24 O'Rourke N, Garcia JC, Paul J et al. A randomised controlled trial of intervention site radiotherapy in malignant pleural mesothelioma. Radiother Oncol 2007; 84:18-22. http://dx.doi.org/10.1016/j. radonc.2007.05.022

\section{CONFERENCING AND EVENTS}

The Royal College of Physicians of Edinburgh has a unique blend of rooms providing the perfect location for your conference, meeting or event. The Victorian Great Hall, galleried New Library and the Georgian Cullen Suite are wonderful settings for dinners and receptions. The modern Conference Centre seats up to 300 people in raked seating and is complemented by breakout rooms seating from 10 to 150 people, a keypad voting system and video conferencing. The College provides a stunning setting for weddings and receptions and is licensed for both civil and religious ceremonies. Discounts are available for Fellows and Members, medical conferences and charities.

For more information or for a quotation, please contact the Events Team on +44 (0) I3। 225 7324; email events@rcpe.ac.uk or visit http://www.rcpe-venue.co.uk 